\title{
Effects of Robot Motion on Human-Robot Collaboration
}

\author{
Anca D. Dragan, Shira Bauman, Jodi Forlizzi, and Siddhartha S. Srinivasa \\ Carnegie Mellon University \\ \{adragan,sbauman,forlizzi,siddh\}@cs.cmu.edu
}

\begin{abstract}
Most motion in robotics is purely functional, planned to achieve the goal and avoid collisions. Such motion is great in isolation, but collaboration affords a human who is watching the motion and making inferences about it, trying to coordinate with the robot to achieve the task. This paper analyzes the benefit of planning motion that explicitly enables the collaborator's inferences on the success of physical collaboration, as measured by both objective and subjective metrics. Results suggest that legible motion, planned to clearly express the robot's intent, leads to more fluent collaborations than predictable motion, planned to match the collaborator's expectations. Furthermore, purely functional motion can harm coordination, which negatively affects both task efficiency, as well as the participants' perception of the collaboration.
\end{abstract}

\section{Categories and Subject Descriptors}

\section{I.2.9 [Artificial Intelligence]: Robotics}

\section{Keywords}

human-robot collaboration; motion; intent; coordination

\section{INTRODUCTION}

This paper studies the role of motion in collaborations between humans and robots, and how planning robot motion that explicitly considers the inferences that the collaborator makes affects the fluency of the collaboration.

Imagine the situation from Fig.1, where a human and a robot collaborate on putting together tea orders. The robot gets the next cup, and the human gathers the corresponding ingredients for it. Now imagine the robot's arm twisting and turning while reaching for the cup, its end effector following the trajectory from the bottom left of Fig.1.

Even though this particular motion is not the most efficient, the robot does get its part of the task done. The motion is functional:

DeFinItion 1.1. Functional motion is motion that reaches the goal and avoids collisions.

Such motion is the main focus of motion planning research [12], and is the state of the art in many robotics applications where robots perform tasks in isolation [17].

Permission to make digital or hard copies of all or part of this work for personal or classroom use is granted without fee provided that copies are not made or distributed for profit or commercial advantage and that copies bear this notice and the full citation on the first page. Copyrights for components of this work owned by others than ACM must be honored. Abstracting with credit is permitted. To copy otherwise, or republish, to post on servers or to redistribute to lists, requires prior specific permission and/or a fee. Request permissions from permissions@acm.org.

HRI'15, March 2-5, 2015, Portland, Oregon, USA.

Copyright (C) 2015 ACM 978-1-4503-2883-8/15/03\$15.00

http://dx.doi.org/10.1145/2696454.2696473.

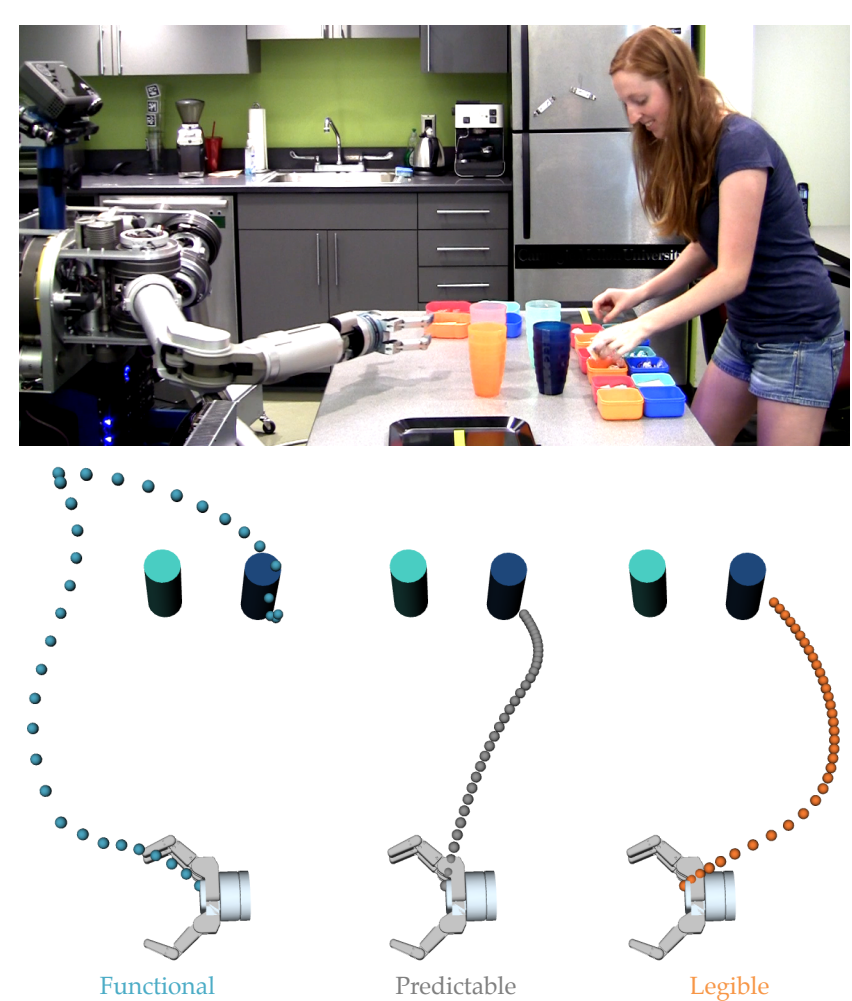

Figure 1: This work manipulates the type of motion the robot plans and studies how this affects physical collaborations between humans and robots.

Collaboration, however, does not happen in isolation. This raises new challenges for the robot's motion, stemming from the two inferences that humans make when they collaborate $[3,20]$ : they infer actions from goals, and goals from actions.

First, the human has an expectation of how the robot will move given the goal it wants to achieve. Motion that matches this expectation is predictable [6]:

Definition 1.2. Predictable motion is functional motion that matches what the collaborator would expect, given the known goal. Fig.1 (bottom center) shows the end effector trace of a predictable motion, which efficiently reaches directly to the goal while avoiding collision with the object.

Second, when the human does not know the robot's goal, he infers the goal given the robot's ongoing motion. Motion that makes this easy is legible [6]:

DeFinition 1.3. Legible motion is functional motion that enables the collaborator to quickly and confidently infer the goal.

Fig.1 (bottom right) shows the end effector trace of the legible motion, which exaggerates the motion to the right to better convey that the goal is the object on the right.

Predictability and legibility are often recognized and studied as important properties of motion $[1,2,6,8,10,14$, 

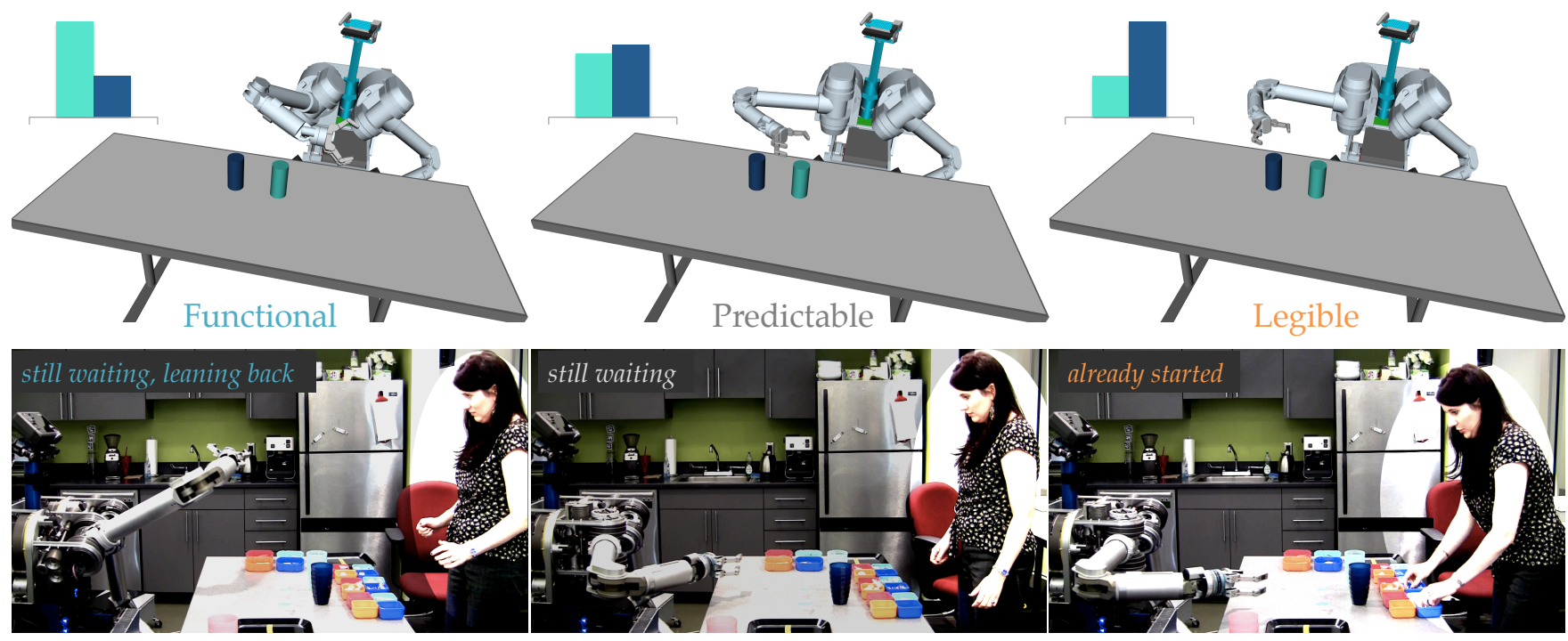

Figure 2: Snapshots from the three types of motion at the same time point along the trajectory. The robot is reaching for the dark blue cup. The functional motion is erratic and somewhat deceptive, and the participant leans back and waits before committing to a color. The predictable motion is efficient, but ambiguous, and the participant is still not willing to commit. The legible motion makes the intent more clear, and the participant is confident enough to start the task.

18]. Our recent work introduced motion planners that autonomously generate motion with these properties, and tested their ability to produce more predictable or more legible motion via online video-based studies in a non-collaborative setting [4,6]. With this, we know the robot can produce more predictable or legible motion, but what we do not know is how this affects human-robot interaction.

This work places all three motion planners - functional, predictable, and legible - in the context of a real physical collaboration in order to test whether the predictability and legibility improvements ultimately affect the collaboration fluency. It uses a task that requires coordinating [15] with the robot (by inferring its goals and performing complementary actions), and study how the choice of a planner affects the fluency of the collaboration through both objective and subjective measures inspired by prior work on fluency [9].

To this end, we designed a study $(N=18)$ with objective measures, like the time it takes for participants to infer their action based on the robot's goal (coordination time), how efficient they are at the task (total task time), and how much they move while the robot is moving (concurrent motion), and subjective measures, like how participants perceive the collaboration in terms of fluency, comfort, trust, etc.

The study revealed that predictable motion was better than functional motion: participants had a significantly easier time working with it, and also perceived it as leading to a significantly more fluent collaboration. Furthermore, because of the coordination required in collaboration [19, 20], legible motion did outperform predictable motion.

These findings support the utility of planning motion for collaboration that goes beyond functionality, and reasons about the collaborator's inferences. However, the study also led to a surprising finding: that participants rationalized the motion. Their perception of predictability and legibility significantly correlated: instead of perceiving one motion as more predictable and the other as more legible, participants perceived whichever motion was easier to coordinate with as also matching their expectations - they rationalized the legible motion as more predictable as well.

What is more, this was solely the effect of doing a task that requires coordination. As our follow-up study suggests, this rationalization no longer occurs when participants do not need to infer the robot's intent. In such cases, participants do perceive the predictable motion as more predictable.

Participants also tended to attribute a lot of agency to the functional motion. They interpreted it as the robot searching through the space, or trying to deceive them.

Overall, this work supports the use of legible motion in collaborative tasks that require coordination, suggesting that collaborators have an easier time coordinating with the robot, subjectively prefer it over predictable motion, and even rationalize it as matching their expectations.

\section{MOTION PLANNERS}

This section summarizes the three motion planning paradigms compared in the study: purely functional motion planning, which is typically achieved via sampling-based methods, and predictable and legible motion planning as defined in our prior work [4], achieved via trajectory optimization.

Notation. The study focuses on goal-directed motion. Here, a robot executes a trajectory $\xi: \mathbb{R} \rightarrow \mathcal{Q}$, lying in a space of trajectories $\Xi$, and mapping time to robot configurations in $\mathcal{Q}$. $\xi$ starts at a configuration $S$ and ends at a goal $G_{R}$ from a set of possible goals $\mathcal{G}$, like the configurations required to grasp the four cups in Fig.1 (top).

\subsection{Functional Motion}

Functional motion solves the classical Motion Planning Problem [12] (also known as the Piano Mover's Problem) of finding a path (an untimed trajectory) from $S$ to $G_{R}$ while avoiding collisions.

For robots with many degrees of freedom, solving this problem requires searching through the high-dimensional space of robot configurations $\mathcal{Q}$. This is commonly achieved through sampling-based planners, among which the RRT [11] is one of the most widely used.

RRTs work by growing a tree rooted at $S$ through the space of free configurations. They do this by sampling configurations and attempting to connect them to the nearest node in the existing tree. Eventually, $G_{R}$ connects to the tree, which 
leads to a collision-free path from $S$ to $G_{R}$. In this work, the functional motion planner applies path-shortening iterations in a post-processing step to eliminate some of the inefficiency of this resulting path.

Fig.1 (bottom left) shows the end effector trace of a functional motion plan to grasp the object on the right. Fig.2 (left) shows a snapshot of the motion, along with a participant's reaction to it. The motion is not efficient, puts the robot in unnatural configurations, and can at times be deceptive about the robot's goal - it might seem like the goal is the one of the left until the very end of the motion.

Thus, we expect that people who collaborate with a robot that produces such motion will not be comfortable, and will not be able to coordinate with the robot because of the difficulty in inferring what the robot is doing.

\subsection{Predictable Motion}

If the collaborator knows that the goal is $G_{R}$, he can predict what trajectory the robot might execute to reach it. The robot's actual trajectory should match this prediction so that the collaborator is comfortable working in the robot's workspace [5]. If it does, then the motion is predictable because the collaborator was able to predict a-priori.

If the collaborator sees the robot as a rational agent, applying the principle of rational action [7], then he expects the robot to be efficient. Efficiency can be modeled via a cost functional

$$
C: \Xi \rightarrow \mathbb{R}_{+}
$$

with lower costs signifying more "efficient" (and thus more expected/predictable to the observer) trajectories. Thus, following [4], the predictable motion planner generates motion via trajectory optimization:

$$
\arg \min _{\xi \in \Xi_{S \rightarrow G_{R}}} C[\xi]
$$

subject to obstacle avoidance requirements which are treated as soft constraints, as in [21].

For the cost $C$, the planner uses the integral over squared velocities, which has been shown to produce predictable trajectories for the type of tasks the robot performs in this work, i.e. tabletop reaching motions [5]:

$$
C[\xi]=\frac{1}{2} \int \xi^{\prime}(t)^{2} d t
$$

Fig.1 (bottom left) shows the end effector trace of a predictable motion plan, a snapshot of which is in Fig.2 (center). This motion is efficient, but it can be ambiguous about the robot's goal, making it difficult to infer its intent. This is especially true in the beginning of the motion, when the predictable trajectory to the goal on the right is very similar to what the predictable trajectory to the goal on the left would look like. The participant in Fig.2 is still waiting to be confident about the robot's intent.

Because predictable motion matches what people expect, we anticipate that people who collaborate with a robot that produces predictable motion will be more comfortable than with functional motion, and better able to coordinate with the robot. However, we expect ambiguous situations to lead to difficulties in coordination, caused by the inability to quickly infer the robot's intent.

\subsection{Legible Motion}

Often times, the collaborator does not know $G_{R}$ a-priori. As he is watching the robot's trajectory, he continually makes an inference as to what the goal of the trajectory might be. The robot's trajectory should enable the collaborator to make the correct inference quickly so that the collaborator be able to easily coordinate with the robot [20]. If it does, then the motion is legible.

Given an ongoing trajectory $\xi_{S \rightarrow Q}$, the probability that the collaborator will assign to any goal $G \in \mathcal{G}$ can be modeled [4] as:

$$
P\left(G \mid \xi_{S \rightarrow Q}\right)=\frac{1}{Z} \frac{\exp \left(-C\left[\xi_{S \rightarrow Q}\right]-V_{G}(Q)\right)}{\exp \left(-V_{G}(S)\right)} P(G)
$$

with $Z$ a normalizer across $\mathcal{G}$ and $V_{G}(q)=\min _{\xi \in \Xi_{q \rightarrow G}} C[\xi]$.

The legible motion planner also generates motion by optimization, much like the predictable planner. However, instead of optimizing $C$, it optimizes the probability that the collaborator will infer $G_{R}$ along the trajectory [4]:

$$
\arg \max _{\xi \in \Xi_{S \rightarrow G_{R}}} \frac{\int P\left(G_{R} \mid \xi_{S \rightarrow \xi(t)}\right) f(t) d t}{\int f(t) d t}
$$

with $f$ a weighting function giving preference to the beginning of the trajectory, when conveying intent is more important. Towards the end, the goal becomes clear with any type of motion.

Fig.1 (bottom right) shows the end effector trace of a legible motion plan, a snapshot of which is in Fig.2 (right). This motion is less efficient than the predictable one (slightly more unpredictable), but, by exaggerating the motion to the right, it more clearly conveys that the actual goal is the one on the right. The participant in Fig. 2 already knows the robot's goal and has started her part of the task in response.

We expect that the benefit of clearly conveying intent will make legible motion better for collaboration than both predictable and functional motion. However, predictable motion is already much better at conveying intent than functional motion is. It is also more predictable (by definition) than legible motion. Together, this can imply a more subtle difference when going from predictability to legibility, than when going from functionality to predictability.

\section{HYPOTHESES}

As the predictions in the previous section suggest, we anticipate that the type of motion the robot plans will affect the collaboration both objectively and subjectively. We also expect it to affect participants' perceptions of how predictable and legible the motions are.

H1 - Objective Collaboration Metrics. Motion type will positively affect the collaboration objectively, with legible motion being the best, and functional motion being the worst.

H2 - Perceptions of the Collaboration. Motion type will positively affect the participants' perception of the collaboration, with legible motion being the best, and functional motion being the worst.

H3 - Perceptions of Legibility and Predictability. Participants will rate the legible motion as more legible than the predictable motion, and the predictable motion as more legible than the functional motion. In contrast, participants will rate the predictable motion as more predictable than the legible motion, and the legible motion as more predictable than the functional motion.

\section{EXPERIMENTAL DESIGN}

To explore the effect of motion type on human-robot collaboration, we conducted a counterbalanced within-subjects study in which participants collaborated on a task with the bimanual mobile manipulator shown in Fig.1. 


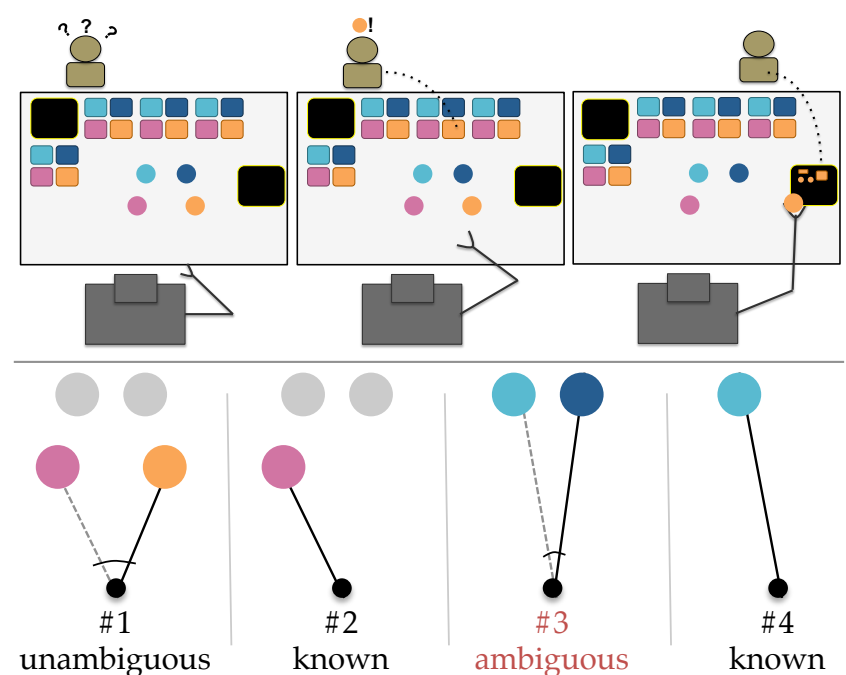

Figure 3: For each tea order, the robot starts reaching for one of the cups. The participant infers the robot's goal and starts gathering the corresponding ingredients. Both place their items on the tray, and move on to the next order. For order \#3, the cups are further away from the robot, and closer to each other, making the situation ambiguous.

\subsection{Task}

Designing a human-robot collaborative task for comparing these types of motion was challenging for four reasons.

First, the success of a collaboration depends on more than the type of robot motion. Other errors during the collaboration can drastically affect the findings. Therefore, the task needs to emphasize the role of motion.

Second, since the study is not testing how the robot should respond to the human's motion, the human's action needs to depend on the robot's, but not vice-versa.

Third, the task must be repeatable: each participant must face the exact same motion planning situations. Different situations (e.g. an object being at a slightly different location) can result in vastly different motions in the case of the functional planner, which could lead to a confound.

And fourth, the task should be as realistic as possible to the participants, and simulate a real world collaboration.

To satisfy these four constraints, the task followed a coffeeshop scenario, in which participants work together with the robot to collaboratively fulfill tea orders. The robot retrieves the correct cup, and the participant gathers the ingredients. Key to this task was that the selection of the ingredients depends on which cup the robot is retrieving.

Fig. 3 shows a schematic of the task setup. There are four orders total, and four different-colored cups. For each order, the robot reaches for one of the cups, and the participant tries to infer the correct color and starts getting the corresponding ingredients from color-coded bins. This emphasizes the role of motion; it does not require that the robot respond to the human; and it leads to a repeatable task because the location of the cups and the order in which the robot picks them up can be predetermined.

The experiment required the participant to fulfill four orders consecutively instead of a single one because (1) this structure places participants in a longer interaction, and (2) it gives participants a chance to familiarize to the motion type. The four orders split into groups of two, as in Fig.3: participants know that the the first two cups the robot reaches for are in the front, and the next two are in the back. Thus, participants do not know the robot's goal a-priori for the first and third order.

The cups are placed such that the situation corresponding to the first order is unambiguous - the cups are far enough apart that the predictable motion should be sufficient to convey the goal early on. The test situation is really the third order, which is ambiguous and thus the best at identifying the differences among the three planners. Furthermore, there is not a strong surprise factor, as each participant will have already seen the robot fulfill two orders.

\subsection{Procedure}

Participants entered the lab and following informed consent, were administered a pre-study questionnaire. Next, the experimenter explained the collaborative task and informed participants that three "programs" were being tested for the robot. They practiced the task once, after which they performed the task three times, one with each "program" (motion type). After each task, they took notes about the collaboration with the robot. At the end, they were administered a post-study questionnaire, and asked to describe the three programs they had experienced.

\subsection{Manipulated Variables}

A single variable, motion type, was manipulated to be functional, predictable, legible. Since the functional planner is nondeterministic, committing to a particular trajectory for each situation is a nontrivial decision. This was done by generating a small set of trajectories and selecting the trajectory with the smallest legibility score. This emphasizes situations where functional motion accidentally leads to deceptive paths, which can harm coordination.

The robot generated predictable and legible trajectories following [4], using functional gradient optimization, and initializing the optimizer with a straight line, constant velocity trajectory. As the next section will detail, one measure is how quickly participants infer the goal. Timing is controlled for by imposing the same duration for all trajectories.

\subsection{Participant Assignment Method}

A total of 18 participants (5 males, 13 females, aged 18$61, M=29.17, S D=12.50$ ) were recruited from the local community. Only five of the participants reported having a technical background.

The experiment used a within-subjects design because it enables participants to compare the three motions. Participants were told that there were three different robot "programs" to avoid biasing them towards explicitly looking for differences in the motion itself.

The order of the conditions was fully counterbalanced to control for order effects. A practice round was used to eliminate some of the variance introduced by the novelty effect. During the practice round, the robot moved predictably, helping to set the predictable motion as their expectation.

The three test rounds (with the three motion types) used the same ordering of the cups, while the practice round used a different ordering. This way, participants would know that the ordering is not set, while allowing for the ability to eliminate cup order as a confound. A single participant noticed the repeating pattern, as detailed in the Analysis section.

\subsection{Dependent Measures}

The measures capture the success of a collaboration in both objective and subjective ways, and are based on Hoffman's metrics for fluency in human-robot collaborations [9]. 
Table I: Subjective Measures

\begin{tabular}{l}
\hline Fluency $\alpha=.91$ \\
1.The human-robot team worked fluently together. \\
2.The robot contributed to the fluency of the team interaction . \\
Robot Contribution [shortened] $\alpha=.75$ \\
1.I had to carry the weight to make the human-robot team better. $(r)$ \\
2.The robot contributed equally to the team performance. \\
3.The robot's performance was an important contribution to the \\
success of the team. \\
Trust $\alpha=.91$ \\
1.I trusted the robot to do the right thing at the right time. \\
2.The robot was trustworthy. \\
3.The robot and I trust each other. \\
Safety/Comfort [extended] $\alpha=.83$ \\
1.I feel uncomfortable with the robot.( $r$ ) \\
2.I believe the robot likes me. \\
3.I feel safe working next to the robot. [new] \\
4.I am confident the robot will not hit me as it is moving. [new] \\
Capability $\alpha=.72$ \\
1.I am confident in the robot's ability to help me. \\
2.The robot is intelligent. \\
\hline Predictability [re-phrased for clarity] $\alpha=.86$ \\
1.If I were told what cup the robot was going to reach for ahead \\
of time, I would be able to correctly anticipate the robot's reaching \\
motion. \\
2.The robot's reaching motion matched what I would have expected \\
given the cup it was reaching for. \\
3.The robot's reaching motion was surprising.( $(r)$ \\
Legibility [new] $\alpha=.95$ \\
1.The robot can reason about how to make it easier for me to predict \\
what it is reaching for. \\
2.It was easy to predict what the robot was reaching for. \\
3.The robot moved in a manner that made its intention clear. \\
4.The robot was trying to move in a way that helped me figure out \\
what it was reaching for. \\
Forced-Choice Questions $\alpha=.91$ \\
1. Which program were you the fastest with? \\
2. Which program was the easiest? \\
3. Which program do you prefer? \\
\hline \hline
\end{tabular}

Objective measures include the coordination time, the total task time, and the concurrent motion time for the test order (order \#3).

The coordination time is the amount of time from the moment the robot starts moving, until the participant infers the correct goal (either by declaring it aloud, which we ask participants to do, or by starting to reach for the correct ingredients, whichever comes first). The total task time is the amount of time, from the moment the robot starts moving, until the last ingredient touches the tray. Finally, the concurrent motion time is the amount of time when both the human and the robot are moving.

Table I shows the seven subjective scales that were used, together with a few forced-choice questions. The fluency and trust scales were used as-is from [9]. The robot contribution scale was shortened to avoid asking participants too many questions. A subset of questions were chosen related to capability, and extended questions were chosen related to safety/comfort. Additional questions were added that were more appropriate to the physical setup (feeling safe next to the robot, and being confident that the robot can avoid collisions with them).

The closeness to the robot question from [16] (not shown in the table) asked participants to select among five diagrams portraying different levels of mental proximity to the robot during the task.
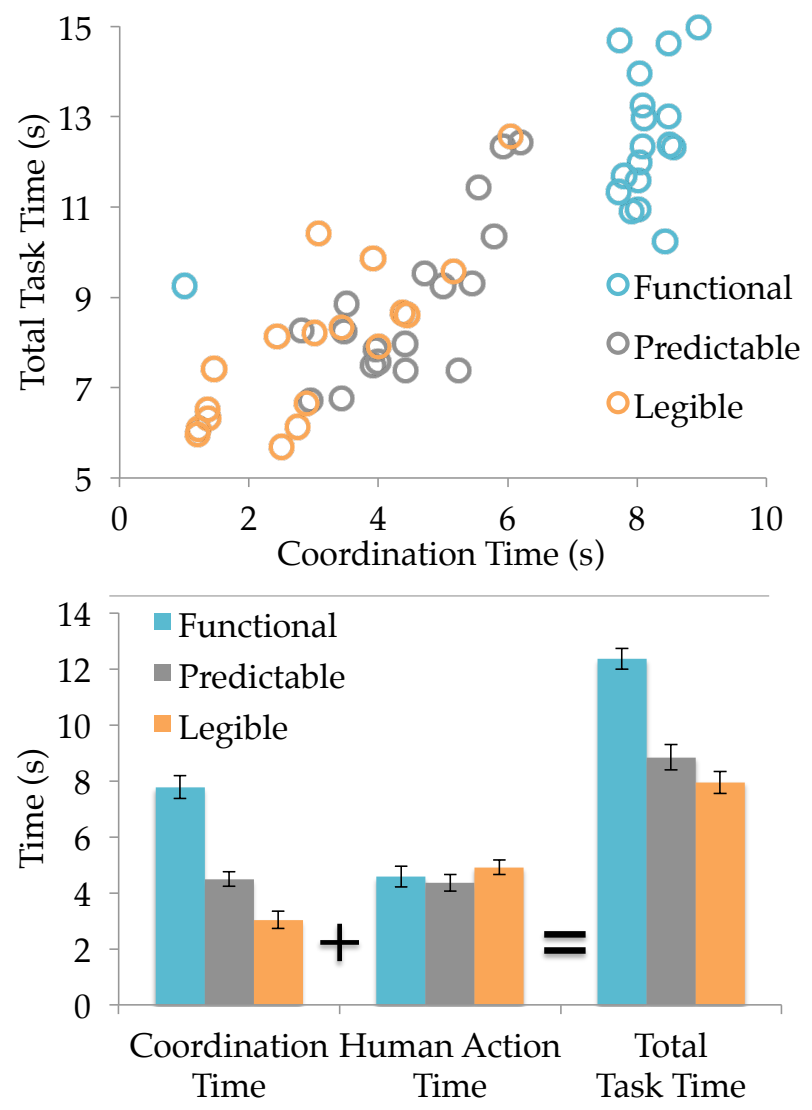

Figure 4: Findings for objective measures.

Additionally, participants answered forced-choice questions at the end, about which program they were the fastest with, which program was easiest to work with, and which program they preferred.

The subjective measures also included perceived predictability and legibility. The predictability scale was adapted from [5]. Clarifications were added because the task was so focused on predicting goals that the word "predictable" was too easily misunderstood in this context.

A legibility scale was devised to capture both how easy inferring the goal is, as well as whether participants believe that the robot has the ability to reason about making this inference easy, and whether it was explicitly trying to do so.

In addition to these measures, a pre-survey was administered to participants, asking demographics questions, as well as the "Big-5" personality questionnaire, since personality type could potentially correlate with how they experience the collaboration.

Finally, the service orientation attitude scale was adapted from [13], measuring whether participants have a relational or utilitarian orientation toward a food service provider. The questions were modified to refer to food preparation. This measure was chosen because having a relational attitude could correlate with the way participants interpret legibility, in particular whether they think the robot is purposefully trying to help them infer the goal easier.

\section{ANALYSIS}

Each of the 18 participants performed the task three times, with each task consisting of four orders (trials). This led to a total of 216 trials, out of which 54 were test trials (order 

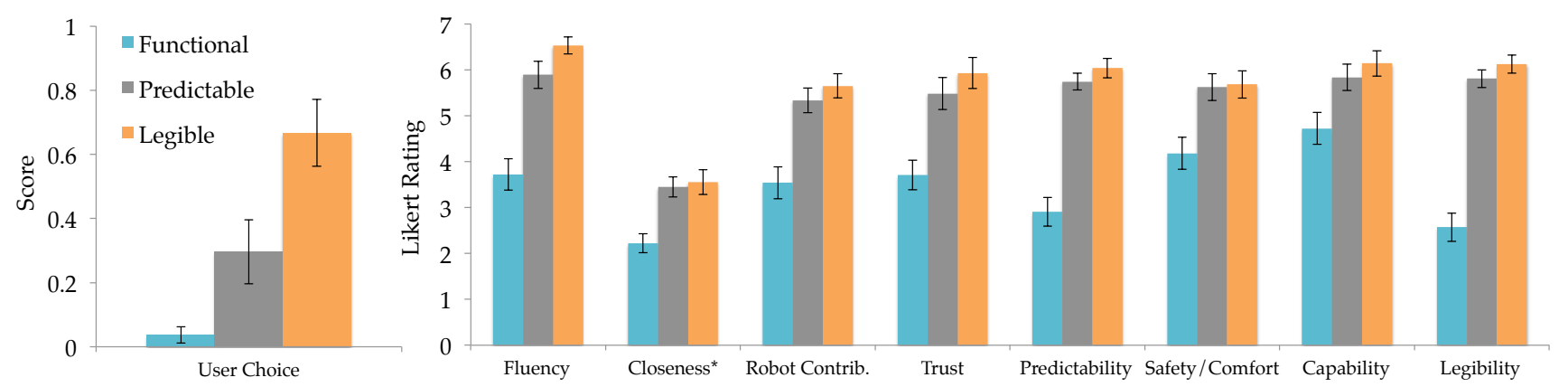

Figure 6: Findings for subjective measures. Closeness was on a 5-point scale.

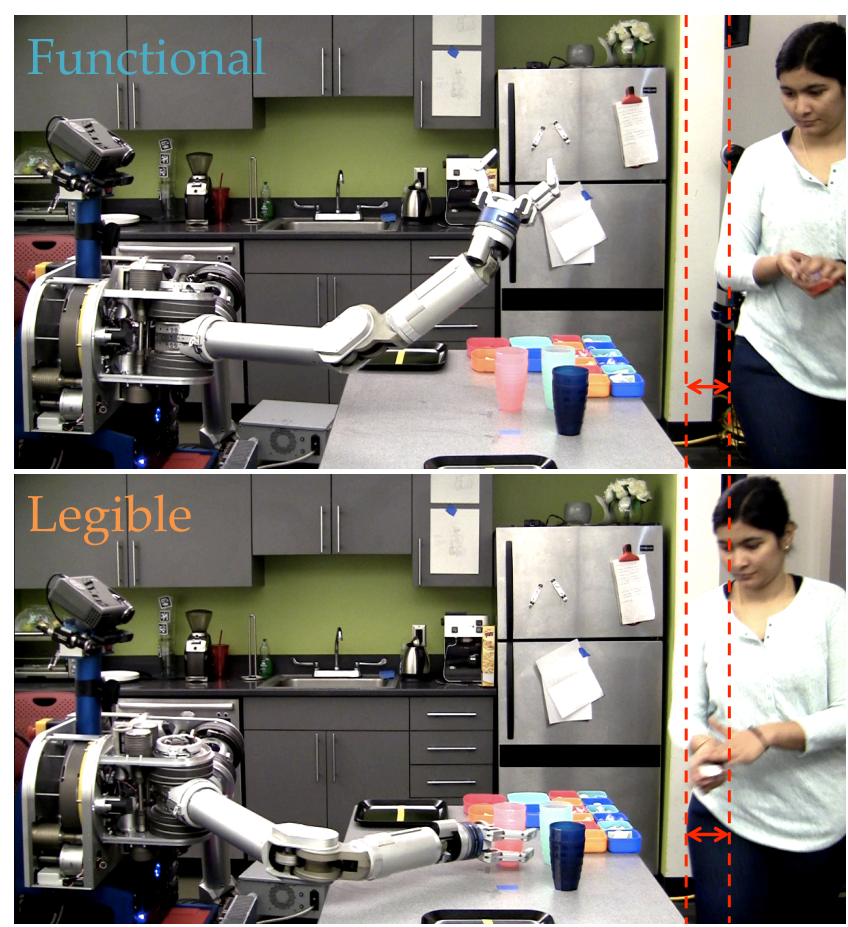

Figure 5: Some of the participants kept a larger distance to the robot during the functional condition. However, most participants were surprisingly comfortable with the robot during this condition.

\#3), 54 were unambiguous trials (order \#1) that still had a coordination time, and the rest were trials that did not need coordination.

\subsection{H1 - Objective Measures}

A repeated measures ANOVA on the coordination time $\left(R^{2}=\right.$ .67) showed a significant effect for motion type $(F(2,51)=$ 52.06, $p<.0001)$, in line with H1.

A post-hoc analysis with Tukey HSD supported H1, showing that all three conditions were significantly different from each other, with functional taking significantly longer than predictable $(p<.0001)$, and predictable taking significantly longer than legible $(p=.01)$. Legible motion resulted in a $33 \%$ decrease in coordination time compared to predictable motion. ${ }^{1}$

\footnotetext{
${ }^{1}$ These results are for the test trials. There was no difference between legibility and predictability on the unambiguous trials (Fig.3), since the predictable motion is sufficiently legible when there is little ambiguity.
}

Fig. 4 shows a scatter plot of the coordination time by the total task time. As expected, legible motion $<$ predictable motion $<$ functional motion in terms of coordination time, with functional motion being better separated as a cluster. These differences propagate to the total task time.

There is one outlier in the plot, for the functional motion (the blue circle in the center left). This was a participant who noticed a repeating pattern in the ordering of the cups, and achieved minimal coordination time as a result during his third condition, which happened to be the functional condition.

A repeated measures ANOVA on the total task time $\left(R^{2}=\right.$ .56) showed similar results. Motion type was significant $(F(2,51)=32.59, p<.0001)$, and the post-hoc showed a significant difference between predictable and functional $(p<.0001)$, partially supporting $\mathbf{H 1}$.

However, the difference between predictable and legible, although trending in the expected direction (Fig. 4 bottom center), was no longer significant ( $p=.27$ ). Surprisingly, participants took slightly longer to gather the ingredients in the legible condition ("human action time", Fig. 4 bottom center). Analysis of the video recordings showed that even though some participants could infer the correct cup earlier, they would hesitate a bit during the task, looking back at the robot again to make sure they made the right prediction and thus slowing down.

Surprisingly, participants did not wait for the robot to finish moving in the functional condition, as we had anticipated. Instead, participants were comfortable enough to do the task while the robot was still moving. Since the robot took longer than the participants to achieve its part of the task, the concurrent motion time was equal to the human action time and did not provide any additional insight.

Participants' main complaint about the functional motion was that it was difficult to coordinate with the robot, and not that they felt unsafe. This could potentially be the result of placing participants in a lab setting, leading to them overtrusting the robot.

Some of the participants did lean back more, as if to avoid the robot arm, and also took a curved path to place the ingredients on the tray (see Fig.5 for an example). Many participants looked surprised when the robot started moving. However, there were some who remained completely unphased by the motion.

Because of the delay in inferring the correct cup, a participant exclaimed "Wait for me!" as she was hurrying to catch up because of the long coordination time. Some of the participants would speed up in gathering the ingredients in the functional condition, as if they were trying to catch up to the robot and still finish the task before. This was not the case in general, with some of the participants having a longer ac- 
tion time than in the predictable condition, stopping more to watch the robot, and hesitating in gathering the ingredients.

None of the participants complained about the robot being much slower than them. This could be due to the bias of participating in a lab experiment. However, as the "Wait for me!" complaint suggests, participants seemed to actually mind the robot finishing its part of the task before they finished theirs, emphasizing the importance of synchronization in collaboration tasks.

Overall, supporting H1, legible motion had significantly lower coordination time than predictable, which had significantly lower coordination time than legible. 17 out of 18 participants had lower coordination time with the legible motion compared to predictable, and 15 had a lower total task time. As expected, the difference between legibility and predictability was more subtle than that between predictability and pure functionality. Surprisingly, the robot moving functionally did not affect concurrent motion time, and participants were comfortable enough to move at the same time as the robot even with functional motion.

\subsection{H2 - Perceptions of the Collaboration}

Table I, which lists the subjective scales, also shows the internal consistency of each scale, reported via Cronbach's $\alpha$. Most scales had good to excellent consistency, the exceptions being capability and robot contribution, which were acceptable. Scale items were combined into a score and analyzed with repeated-measures ANOVAs. Fig.6 plots the results.

The score produced by the overall forced-choice questions was significantly affected by the motion type $(F(2,51)=$ $13.59, p<.0001)$, with the post-hoc revealing that legible motion had a significantly higher score than predictable motion $(p<.01)$, but predictable motion was only marginally better than functional motion $(p=.08) .12$ out of the 18 participants preferred the legible motion.

All the Likert ratings showed a significant effect for motion type as well, with post-hocs revealing that functional motion was significantly lower rated than predictable and legible motion in every case (with $p<.0001$, except for capability, details below). The legible motion tended to be rated higher than predictable, but those differences were not significant. Fig.6 summarizes these findings.

The biggest difference between predictability and legibility was in fluency. Safety, on the other hand, was the same for both - this is not surprising, given that legible motion is better at conveying intent, but this does not necessarily lead to an increased feeling of safety.

Capability was high with the functional motion as well, though still significantly lower than with predictable motion $(p=.03)$.

With respect to additional participant measures, unsurprisingly, being extroverted significantly correlated to having a relation attitude towards a food preparation partner $(r(16)=.51, p=.03)$. Additionally, extroversion inversely correlated with preferring the legible motion over the other two motion types $(r(16)=-.49, p=.04)$. However, extroversion did not correlate with whether or not the legible motion worked objectively, i.e. achieved lower coordination time. More research is needed to verify this result and understand why introverts might be more likely to appreciate a legible robot.

Overall, participants significantly preferred the legible motion over the predictable motion, and tended to prefer the predictable motion over the functional. However, as with the objective measures, their ratings of the collaboration sug- gest that legibility is a more subtle improvement over predictability, compared to the improvement of predictability over functionality.

\subsection{H3 - Perceptions of Predictability and Legibility: Rationalization of the Motion}

\subsubsection{Perceptions of Legibility}

As predicted by $\mathbf{H} 3$, motion type significantly affected the legibility rating $(F(2,51)=67.56, p<.0001)$. The posthoc analysis did show a significant difference between functional and predictable motion $(p<.0001)$, but not between predictable and legible motion.

The biggest difference between predictable and legible motion was in how easy participants thought it was to predict the robot's goal (question 2) (mean 6 vs. 6.61). Participants thought the legible motion made goal inference easier. In contrast, participants did not think that the robot was more capable of higher-order reasoning. Question 1 yielded almost no difference between predictability and legibility, and had a lower overall mean (5.11 vs. 5.27).

Participants' comments matched their ratings of legibility of motion. Three participants described the functional motion as "exaggerated", with one of them commenting that "the arm motions were so exaggerated that it was hard to see which cup he was going to choose until just before". Many of the participants referred to it as less intent-expressive, commenting that "it made it almost impossible to guess" or that it was "trickier".

One participant said that the functional motion made her less confident about the intent even for the orders where the cup was predetermined (2nd and 4th): "even when I knew the cup it would grab, I was still less confident than with the other programs". Indeed, we noticed some participants hesitate more during the functional motion condition on these orders, while others remained completely focused and ignored the erratic nature of the motion.

Interestingly, some participants attributed agency to the random nature of the functional motion: "he was picking a cup at random", "the robot appeared to be searching before selecting a cup", "makes me think that it's playing on purpose", "it appeared that the robot had a mind of its own, along with its own agenda", the robot "tricked me". One participant actually rated the functional program as the one they prefer overall, and a couple rated it as the most intelligent of the three, possibly because of this attribution of agency.

Because the predictable and legible motions are more similar to each other than they are to the functional motion, participants tended to contrast the two in their descriptions of the three programs.

Most participants described the predictable motion as somewhat less intent-expressive than the legible: "slightly harder to recognize", "the direction it's going in isn't as clear as the (legible motion)", "slight uncertainty about the cup choice", "not very clear as the (legible motion)", "not as easy as (the legible motion); I had to wait a bit after his hand moved to realize the cup he was going for", "it was had to determine which he'd pick", "it was not as clear".

In contrast, the descriptions for the legible motion referred to it as "easier to predict [the cup]" and "very straightforward", noting that one "could clearly see the trajectory of its hand to the cup". Some of the participants recognized that the robot was altering the motion in order to better convey intent. They thought that "the wide movements made it easy to identify [the cup]", "the angle was such that you 
could discern", and that "he starts out clearly moving towards one direction".

One of the participants even associated the beginning of the robot's legible motion to a communicative gesture: "it was almost like the robot was pointing at the cup he was going for right before, while he was moving his arm".

\subsubsection{Perceptions of Predictability}

Motion type significantly affected the predictability rating as well $(F=50.48, p<.0001)$. Counter to $\mathbf{H 3}$, however, participants actually tended to rate the legible motion higher, and the ratings for predictability and legibility significantly correlated $(r(52)=.91, p<.0001)$.

It appears that when legibility works for someone and they can infer the goal easier, they tend to rationalize it as the "natural" motion, or even "direct" or "efficient". In contrast, some participants refer to the predictable motion as "inefficient", and even as "going towards the other cup initially", which is inaccurate.

This rationalization may happen because of the importance of inferring intent in the task. Legible motion is easier for collaboration, and that makes participants believe it is what they would have expected.

\subsubsection{Follow-Up Study on Predictability}

To test this, an online follow-up study $(N=16)$ was conducted, where participants were shown a motion from the practice round, and asked to think about the motion they expect in the test situation. They then watched a video of each motion (predictable and legible), and chose which better matched their expectation. Since the task no longer had an intent prediction emphasis, participants did choose the predictable motion significantly more often (approx. $70 \%$, which a binomial test showed to be significantly higher than chance, i.e. $50 \%, p=.0251$ ). This supports our rationalization hypothesis.

Overall, perceptions of predictability and legibility correlate in a task in which intent inference is important: participants rationalize the motion that makes the coordination easier as the motion they think they would expect.

\section{DISCUSSION}

Overall, the findings from this study suggest that functional motion is not enough for collaborative tasks that require coordination, and that the robot should take the collaborator's expectations into account when planning motion. Although this was a laboratory study with an artificial task, the findings lead to interesting conjectures about motion design for collaborative tasks.

One finding is that legibility is preferable to predictability in coordination tasks, as it decreases coordination time, collaborators prefer it overall, and rationalize it as more predictable despite it actually being less efficient (and them not being able to anticipate it a-priori). Furthermore, for quadratic costs $C$, legibility has no computational overhead compared to predictability in planning time.

Furthermore, functional motion might be enough for tasks that do not require coordination nor close proximity (such as repetitive tasks like those one might encounter on a factory floor, or tasks that have been carefully planned in advance, with separate and known roles). Participants were surprisingly willing to move at the same time as the robot, and mainly complained about not being able to coordinate. Furthermore, functional motion does not require optimization, making it at times faster at producing a feasible plan [21].
Predictable motion seems to be best when coordination is not necessary (or the situations are not ambiguous, making the predictable motion legible enough), but when people work in close proximity to the robot and would be uncomfortable with surprising motion [5].

\section{CONCLUSION}

This paper analyzed the benefit of planning robot motion that explicitly enables the collaborator's inferences on the success of a physical collaboration. Results suggest that legible motion, planned to clearly express the robot's intent, leads to more fluent collaborations than predictable motion, planned to match the collaborator's expectations. Functional motion was found to negatively affect coordination, increasing the time it takes to achieve the task, as well as the participant's perception of the collaboration.

Future work will explore how people change their expectations of motion over time (e.g. does legible motion become objectively predictable?). The effect of multi-modal legibility for coordination will also be studied, to understand when the robot should express intent through motion, and when it should speak, or even gesture.

\section{References}

[1] R. Alami, A. Albu-Schaeffer, A. Bicchi, R. Bischoff, R. Chatila, A. D. Luca, A. D. Santis, G. Giralt, J. Guiochet, G. Hirzinger, F. Ingrand, V. Lippiello, R. Mattone, D. Powell, S. Sen, B. Siciliano, G. Tonietti, and L. Villani. Safe and Dependable Physical Human-Robot Interaction in Anthropic Domains: State of the Art and Challenges. In IROS Workshop on $p H R I, 2006$.

[2] R. Alami, A. Clodic, V. Montreuil, E. A. Sisbot, and R. Chatila. Task planning for human-robot interaction. In Smart objects and ambient intelligence, 2005.

[3] G. Csibra and G. Gergely. Obsessed with goals: Functions and mechanisms of teleological interpretation of actions in humans. Acta Psychologica, 124(1):60 - 78, 2007.

[4] A. Dragan and S. Srinivasa. Generating legible motion. In RSS, 2013.

[5] A. Dragan and S. Srinivasa. Familiarization to robot motion. In HRI, 2014.

[6] A. D. Dragan, K. C. Lee, and S. S. Srinivasa. Legibility and predictability of robot motion. In HRI, 2013.

[7] G. Gergely, Z. Nadasdy, G. Csibra, and S. Biro. Taking the intentional stance at 12 months of age. Cognition, 56(2):165 - 193, 1995.

[8] M. J. Gielniak and A. L. Thomaz. Generating anticipation in robot motion. In RO-MAN, 2011.

[9] G. Hoffman. Evaluating fluency in human-robot collaboration. In HRI Workshop on Human Robot Collaboration, 2013.

[10] T. S. Jim Mainprice, E. Akin Sisbot and R. Alami. Planning safe and legible hand-over motions for human-robot interaction. In IARP Workshop on Technical Challenges for Dependable Robots in Human Environments, 2010.

[11] J. J. Kuffner and S. M. LaValle. Rrt-connect: An efficient approach to single-query path planning. In ICRA, 2000.

[12] J.-C. Latombe. Robot Motion Planning. Kluwer Academic Publishers, Norwell, MA, USA, 1991.

[13] M. K. Lee, S. Kiesler, J. Forlizzi, S. Srinivasa, and P. Rybski. Gracefully mitigating breakdowns in robotic services. In HRI, 2010.

[14] C. Lichtenthäler, T. Lorenz, and A. Kirsch. Towards a legibility metric: How to measure the perceived value of a robot. In ICSR Work-In-Progress-Track, 2011.

[15] M. A. Marks, M. J. Sabella, C. S. Burke, and S. J. Zaccaro. The impact of cross-training on team effectiveness. Journal of Applied Psychology, 87(1):3, 2002.

[16] B. Mutlu, J. Forlizzi, and J. Hodgins. A storytelling robot: Modeling and evaluation of human-like gaze behavior. In Humanoid Robots, 2006.

[17] I. Sucan, M. Moll, and L. Kavraki. The open motion planning library. Robotics \& Automation Magazine, IEEE, 2012

[18] L. Takayama, D. Dooley, and W. Ju. Expressing thought: improving robot readability with animation principles. In HRI, 2011.

[19] M. Tomasello, M. Carptenter, J. Call, T. Behne, and H. Moll. Understanding and sharing intentions: the origins of cultural cognition. Behavioral and Brain Sciences, 2004

[20] C. Vesper, S. Butterfill, G. Knoblich, and N. Sebanz. A minimal architecture for joint action. Neural Networks, 23(8):998-1003, 2010

[21] M. Zucker, N. Ratliff, A. Dragan, M. Pivtoraiko, M. Klingensmith, C. Dellin, J. Bagnell, and S. Srinivasa. Covariant hamiltonian optimization for motion planning. IJRR, 2013. 